\title{
The Relevance of a Back-Scatter Model for Depth-A veraged Flow Simulation
}

\author{
Bram C. van Prooijen • Wim S. J. Uijttewaal
}

Received: 14 August 2007 / Accepted: 11 June 2008 / Published online: 15 August 2008

(C) The Author(s) 2008

\begin{abstract}
This study demonstrates the importance of a sophisticated sub-grid model when performing a depth-averaged unsteady RANS simulation of a shallow flow. The reduction of resolution and the spatial dimensions exclude important physical processes as present in three-dimensional turbulence. Especially the effect of the bottom turbulence on the formation of horizontal eddies appears of key importance. A method is proposed to incorporate these effects by means of a kinematic simulation that mimics the residual turbulent fluctuations in a straight channel flow after depthaveraging. This method is developed in the context of the evolution of large eddies in a shallow mixing layer. A comparison with experiments shows that the proposed method works satisfactory. Naturally, it does not fully account for the omission of all 3D-effects.
\end{abstract}

Keywords Open channel $\cdot$ Large eddy simulation $\cdot$ Shallow flow $\cdot$ Mixing layer

\section{Introduction}

The promising developments with respect to applications of Large Eddy Simulation (LES) to small scale industrial flows, has stimulated the use of LES also for complex environmental flows. However, the typically high Reynolds numbers and

\footnotetext{
B. C. van Prooijen

Hydraulic Engineering Section, Faculty of Civil Engineering and Geosciences,

Delft University of Technology, P.O. Box 5048, 2600 GA,

Delft, The Netherlands

e-mail: b.c.vanprooijen@tudelft.nl

W. S. J. Uijttewaal $(\varangle)$

Environmental Fluid Mechanics Section, Faculty of Civil Engineering and Geosciences,

Delft University of Technology, P.O. Box 5048, 2600 GA,

Delft, The Netherlands

e-mail: w.s.j.uijttewaal@tudelft.nl
} 
complex, dynamically changing flow domain exclude a straightforward adoption of the developed methods. Taking into account the large but limited growth in computer capacity, it becomes clear that a full 3D LES simulation of an estuarine flow with a typical Reynolds number, $R e=10^{7}$ will not be feasible in the next few decades. We therefore have to take refuge in simplified modelling that allows for a strong reduction of the dimension of the computational grid and consequently of the resolution.

In the natural environment many flows may be considered as shallow. The vertical length scale in rivers, lakes and coastal regions is confined by the bottom and free surface and is usually orders of magnitude smaller than the horizontal length scales. The latter contribute most to the horizontal transfer of momentum thereby governing the overall flow pattern. The anisotropy of the large scale motion generally gives rise to quasi two-dimensional characteristics which are rather uniform along the vertical coordinate $[27,32]$. Most features of such a flow are therefore expected to be captured by a depth-averaged model $[13,14,34]$.

With every kind of averaging and filtering information is lost. This lost information should be replaced by extra terms in the momentum and energy balances. With depth-averaging the complex processes that take place in the high velocity gradient near the bottom are usually lumped in friction coefficients and effective turbulent diffusivity. The remaining two-dimensional problem has little to do with the original one because it has become a kind of laminar flow where much of the dynamics is filtered out. Especially when the bulk flow is essentially unstable due to horizontal shear the turbulent kinetic energy necessary to trigger the formation of large horizontal structures is lacking in a depth-averaged approach.

\subsection{The shallow mixing layer}

A simple but interesting type of flow in this respect is the shallow mixing layer. This flow consists of two shallow streams that form a shear layer in a horizontal plane downstream of the merging point, with the velocity gradient perpendicular to the vertical plane between the streams. Such a shallow mixing layer is typically found at the merging of two rivers. The instabilities in the shear layer give rise to the formation of flow structures (with vertical vorticity axes) much larger than the water depth that move in the horizontal plane confined by the no-slip bottom and the free-slip surface, see Fig. 1. These coherent structures are referred to as quasi $2 \mathrm{D}$ turbulence in this paper.

The bottom boundary layer is fully turbulent with three-dimensional coherent structures. The associated dominant length scale is in the order of magnitude of the water depth and therefore (much) smaller than the dominant length scale of the quasi $2 \mathrm{D}$ turbulence. We refer to 3D bottom turbulence in this paper.

The resulting flow thus contains a range of length scales that covers several orders of magnitude. Although the scales of the 3D bottom turbulence and the quasi $2 \mathrm{D}$ turbulence are rather disparate, the energy balance of the turbulence is governed by both. Previous studies on shallow shear flows $[5,7,31]$ have indicated that the dynamics of the quasi $2 \mathrm{D}$ turbulence in combination with $3 \mathrm{D}$ turbulence is not well understood and difficult to represent in an efficient model. 
Fig. 1 Perspective view on the visualisation of a shallow mixing layer in a laboratory flume, $3 \mathrm{~m}$ wide, $67 \mathrm{~mm}$ deep, showing large eddies up to $1 \mathrm{~m}$ diameter and the small-scale bottom turbulence [34]. The arrows indicate the mean streamwise velocity

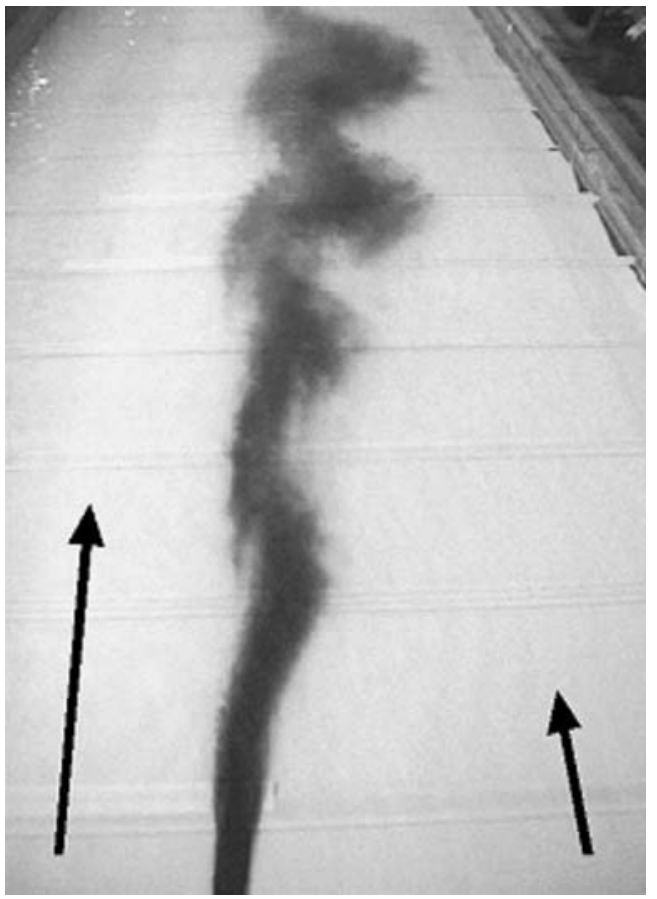

\subsection{Linear behaviour of unstable flows in a shallow mixing layer}

A study by [33] has revealed that the spectral distribution of the large scale motions of a shallow mixing layer can be predicted from a linear stability analysis. First, the mean flow was predicted, based on the self-similarity method proposed by [5]. This resulted in the transverse profiles of the mean streamwise velocity. Secondly, these profiles were substituted into a linear stability analysis, resulting in amplification factors for each frequency at each position downstream of the beginning of the mixing layer. Thirdly, these amplification factors were integrated in downstream direction, leading to the amplification factors of the inflow perturbations. Finally, the energy density spectra at each position downstream could be obtained by imposing a proper spectrum for the inflow perturbations. This spectrum represents the quasi 2D turbulence in a uniform channel flow after depth averaging (see Section 2.3 for further explanation) and is referred to as background quasi $2 \mathrm{D}$ turbulence. The spectrum of the inflow perturbations was assumed to be constant for all frequencies, see Fig. 2. The resulting energy density spectra at several downstream positions are shown in Fig. 2 as well. It should be noted that the energy density level at low frequencies is for one-dimensional spectra affected by aliasing due to oblique wave vectors [30]. For the triggering of large scale instabilities also the projected wave length plays a role.

A straightforward consequence of this work is the notion that the properties of long-living flow structures are determined by the up-stream flow properties from which they have emerged. This implies also that the intensity level from which the 

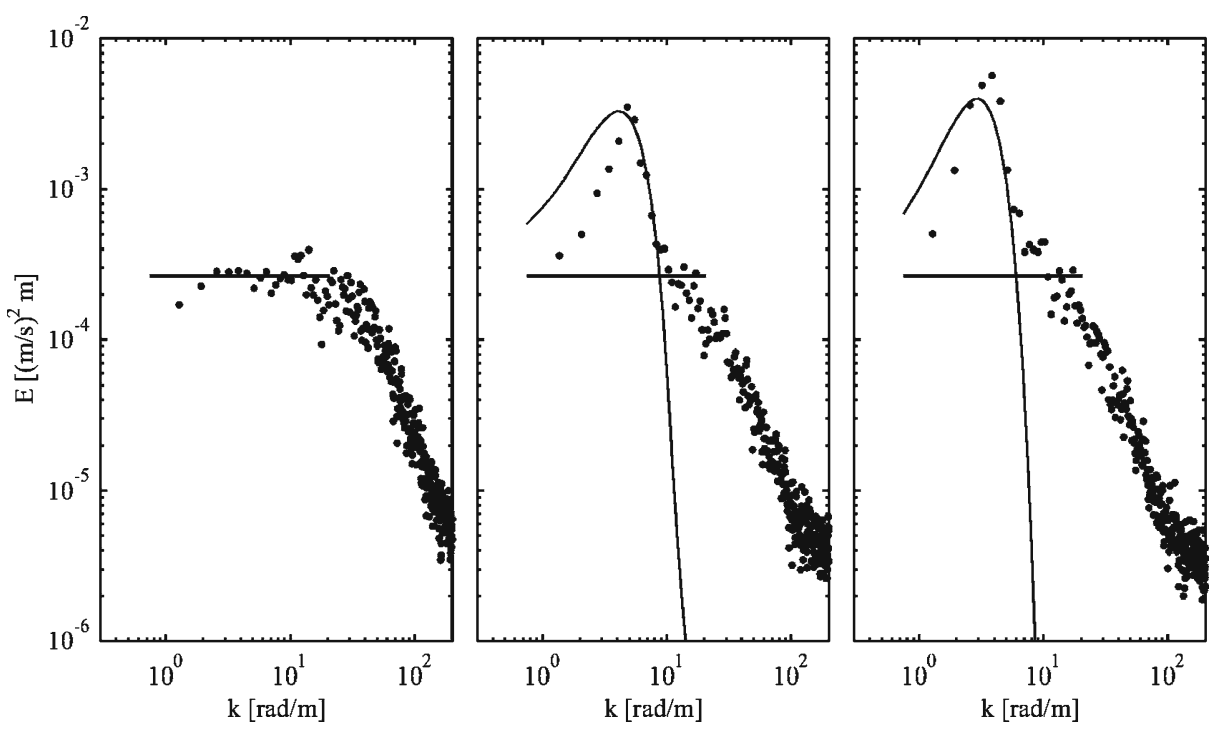

Fig. 2 Spectral intensity distributions of the transverse velocity component at three downstream positions of a mixing layer with $67 \mathrm{~mm}$ water depth: $0 \mathrm{~m}$ (left), $4.5 \mathrm{~m}$ (middle) and $10 \mathrm{~m}$ (right). Values measured at the free-surface (dots) are compared with a prediction derived from a linear stability analysis (lines). The straight horizontal line represents the uniform energy level imposed at the inflow [33]

structures start to grow are of key importance for their strength. The turbulence properties of the ambient flow therefore play an important role in the energy transfer between the turbulent scales in the mixing layer and should thus be accounted for.

\subsection{The need for representing unresolved motion}

The above example shows that the coupling between the instability mechanism and the background quasi 2D turbulence is needed to estimate the proper energy density levels. Extending this line of thought suggests the existence of other cases where re-introducing the dynamics into the simulation via some kind of backscatter mechanism will be important. A clear example is found in the developments towards hybrid RANS-LES methods. Their performances can benefit from prescribing a proper guess of the time dependent turbulent fluctuations that are present near boundaries or at the sub-grid level. A number of different applications for which such a model extension can be useful are described below.

\subsubsection{As inflow condition}

With LES and DNS periodic boundary conditions, as often applied to simple geometries, can not be used for spatially developing flows and the inflow boundary therefore needs to represent the upstream turbulence properties in a realistic manner. This is best done by generating the upstream turbulence in a separate simulation of a periodic straight channel flow [19]. In order to avoid this rather expensive method the dynamics of the in-flowing turbulence can be represented by a kinematic velocity 
field. With the proper prescription of turbulent kinetic energy, length and time scales the inflow conditions can be made rather realistic saving substantial computational efforts [17].

\subsubsection{Representing subgrid dynamics}

In a simulation with a coarse resolution a significant part of the turbulence spectrum is lumped in the sub-grid model. The dissipative character of most sub-grid models does not allow energy to be transferred from sub-grid scales to resolved scales. Such a backscatter of turbulent kinetic energy is particularly relevant in boundary layers and other regions of high shear, and leads to better simulation results when accounted for $[4,6,20]$. In case of the transport of e.g. a passive tracer, the dynamics on the subgrid scale will contribute to the mixing in a way that can not be modelled as diffusion since the real process is advection by the small scales. This has consequences for the statistics of two-particle dispersion, chemical reactions and other processes where the small scale kinetics needs to be separated from diffusion [10].

\subsubsection{As boundary condition}

Many applications with LES make use of wall functions [22, 26], or a two-layer approach $[1,12,29]$. This has clear advantages with respect to computational demands. No grid refinement near the wall is needed and the computational time step can remain large. The complex dynamics that occurs in the near-wall region with its high velocity gradient is however not resolved and at best estimated as a local turbulent kinetic energy level. In order to account for the turbulent fluctuations that are generated, a coupling needs to be made between the non-resolved turbulence in the near-wall region and the time dependent fluctuations as they are resolved by the LES [16]. When the resolution is such that a sufficient part of the ejection and sweep structures are resolved no specific measures need to be taken [22]. Recent work by [23] confirms the idea that a backscatter model is needed which is forcing velocity fluctuations at the interface between the RANS near wall model and the LES in the outer layer $[20,35]$.

\subsubsection{Representing subgrid dynamics in depth-averaged modelling}

The applications described above will be of particular importance when the reduction of the problem goes as far as depth-averaged modelling. In the case considered here all information along the vertical coordinate is lost and its effect on the horizontal motion needs to be replaced. Moreover the remaining two-dimensional problem will exhibit the characteristic energy transfer to smaller as well as to larger length scales [2]. Special care should therefore be taken of the resolved turbulent kinetic energy and associated energy cascades. The resolution of the remaining twodimensional model should at least be of the order of the water depth, so little more than the large scale anisotropic motion can be resolved. As demonstrated above with the example of the mixing layer, the onset of shear instabilities is governed by the background quasi 2D turbulence level. The latter cannot be represented in a depth-averaged simulation as it is a result of the $3 \mathrm{D}$ bottom turbulence. In a depthaveraged simulation of a uniform channel all fluctuations will dampen, as there is no production due to the lack of a transverse velocity gradient. The background 
quasi 2D turbulence thus needs to be mimicked in an efficient way by means of a backscatter model.

In order to obtain a clear view on the role of the forced fluctuations we will start with an example of the depth-averaged modelling of a shallow mixing layer with forcing at the inflow boundary. This can be considered as the non-linear implementation of the above described linear stability analysis [33]. Second, a backscatter model for the background quasi 2D turbulence will be described that includes the characteristics of large turbulence structures as they appear in a uniform open channel flow. These characteristics are obtained from a careful analysis of a 3D-LES of a wide open channel. The back-scatter model will be forcing the whole domain in the form of kinematic simulation. Third, the model will be applied to the depth averaged simulation of shallow mixing layers.

\section{Depth Averaged Modelling}

\subsection{Model set-up}

In the shallow mixing layer the large scale features move predominantly in the horizontal plane and can therefore be captured with a relatively small computational effort in a depth averaged formulation with a stress-free rigid lid assumption. After depth-averaging the continuity and Navier-Stokes equations result in:

$$
\begin{gathered}
\frac{\partial \tilde{u}}{\partial x}+\frac{\partial \tilde{v}}{\partial y}=0, \\
\frac{\partial \tilde{u}}{\partial t}+\tilde{u} \frac{\partial \tilde{u}}{\partial x}+\tilde{v} \frac{\partial \tilde{u}}{\partial y}=-\frac{1}{\rho} \frac{\partial \tilde{p}}{\partial x}-\frac{c_{f}}{D} \tilde{u} \sqrt{\tilde{u}^{2}+\tilde{v}^{2}}+v_{h o r} \nabla^{2} \tilde{u}, \\
\frac{\partial \tilde{v}}{\partial t}+\tilde{u} \frac{\partial \tilde{v}}{\partial x}+\tilde{v} \frac{\partial \tilde{v}}{\partial y}=-\frac{1}{\rho} \frac{\partial \tilde{p}}{\partial y}-\frac{c_{f}}{D} \tilde{v} \sqrt{\tilde{u}^{2}+\tilde{v}^{2}}+v_{h o r} \nabla^{2} \tilde{v} .
\end{gathered}
$$

The tilde $\sim$ identifies short-time and depth averaged properties such that all smallscale (3D) turbulence can be represented by an effective horizontal eddy viscosity. $D$ denotes the constant water depth, $p$ the pressure, $u$ and $v$ the horizontal velocities in streamwise $x$-direction and transverse $y$-direction respectively, $c_{f}$ the bottom friction parameter and $v_{\text {hor }}$ the turbulence viscosity that acts in the horizontal plane and is caused by the non-resolved bottom turbulence. Assuming fully developed boundary layers in the vertical direction the eddy viscosity can be estimated using a standard Elder formulation,

$$
v_{\text {hor }}=\alpha_{\text {hor }} u \times D,
$$

The constant $\alpha_{\text {hor }}$ still needs to be chosen. A direct measurement of this coefficient is difficult to obtain but the associated value for $\alpha_{\text {hor }}$ is in the range of $0.1-0.2$. Following Elder [8], the ratio between the eddy diffusivity and eddy viscosity (Prandtl-Schmidt number) turned out to be approximately 1 for vertical mixing, (see e.g. [9]). It is therefore assumed that this also applies for the Prandtl-Schmidt number for the horizontal mixing. Therefore $\alpha_{\text {hor }}=0.15$ has been adopted. This value is higher 
than the value of 0.067 derived by Elder [8] for the vertical mixing only. This is motivated by the higher turbulence intensities and larger length scales associated with the motion in the horizontal plane than in the vertical [9]. In recent work by and Hinterberger and Rodi [15] a smaller value for the horizontal eddy viscosity was used, based on 3D-LES, choosing the vertical and horizontal eddy viscosities equal $\left(\alpha_{\text {hor }}=\alpha_{\text {ver }}=0.067\right)$ and using a Prandtl-Schmidt number of 0.7 for the horizontal diffusivity. This resulted in an effective horizontal diffusion of $0.1 u \times D$, which is at the lower end of experimental observations.

With the closure used here the set of equations can be solved numerically, provided a proper implementation of the in- and outflow conditions. The time dependent solution allows for the formation of large eddy structures in case the resolution is sufficient to represent them and the mechanism by which they are formed. Even with a resolution of the order of the water depth this approach should formally be labelled 2D-URANS (unsteady Reynolds averaged NavierStokes), rather than LES, because the turbulence closure applies to all scales and is thus not grid dependent. Furthermore the $2 \mathrm{D}$-approach prohibits a solution that includes a part of the inertial sub-range, violating the standard assumptions with LES: homogeneity and isotropy at the sub-grid scale level [24, 28].

\subsection{Shallow mixing layer with inflow perturbations}

The experiments as described in [33] and [34] are simulated. The simulation covers a domain of $3 \mathrm{~m}$ width and $17 \mathrm{~m}$ length, and a water depth $D=0.067 \mathrm{~m}$. The uniform grid of $150 \times 850$ cells results in a mesh size of $\Delta x=\Delta y=0.02 \mathrm{~m} \approx 0.3 D$. Freeslip conditions are imposed at the side boundaries and at the surface. An advective condition is imposed at the outflow boundary. With respect to the mean flow a hyperbolic tangent profile is imposed at the inflow boundary corresponding to a mixing layer width equal to the water depth $\delta\left(x_{0}\right)=D$ and a velocity difference equal to the experimental value of $0.19 \mathrm{~m} / \mathrm{s}$. The splitter plate itself is thus not represented in the model.

Although discretisation noise will eventually trigger the shear instabilities in the mixing layer that lead to the large eddy structures, the amplitude of the noise was shown to be of paramount importance for the intensity of those structures as they develop along the mixing layer, Fig. 2. Obviously the amplitude needed for an appropriate representation has to correspond with the background quasi $2 \mathrm{D}$ turbulence as it is generated in the bottom boundary layer. For the two velocity components at the inflow boundary a perturbation is imposed with a uniform spectrum for the low frequency part, in accordance with the spectral distribution of a straight channel flow; see also Fig. 2. The perturbations are built from a set of 200 Fourier modes, in such a way that continuity is obeyed. More details of the inflow perturbations will be explained later on, see also [34].

In Fig. 3 the evolution of the vortex structures in the mixing layer is shown by means of the Weiss-function. The dark areas indicate dominance of vorticity. In the upper panel only little effect is seen at the very beginning of the mixing layer. In the absence of inflow perturbations the mixing layer remains smooth and does not show any structures. In the presence of a constant pressure gradient for the whole domain the bottom friction tends to homogenize the flow resulting in a decreasing velocity difference in downstream direction. As a consequence of mass conservation 
Fig. 3 The effect of perturbation amplitude on the genesis of vortex structures in the simulation of a depth averaged shallow mixing layer. The flow is from left to right with the high velocity introduced at $y>0$ and low velocity at $y<0$. The Weiss-function (squared shear minus squared vorticity) is plotted for the cases: with no perturbations (top), small amplitude (with spectral energy density $E_{\text {per }}=$ $0.01 \mathrm{c}_{f} \mathrm{U}^{2} \mathrm{D}$ middle), high amplitude (with spectral energy density $E_{\text {per }}=$ $1.0 \mathrm{c}_{f} \mathrm{U}^{2} \mathrm{D}$ bottom)

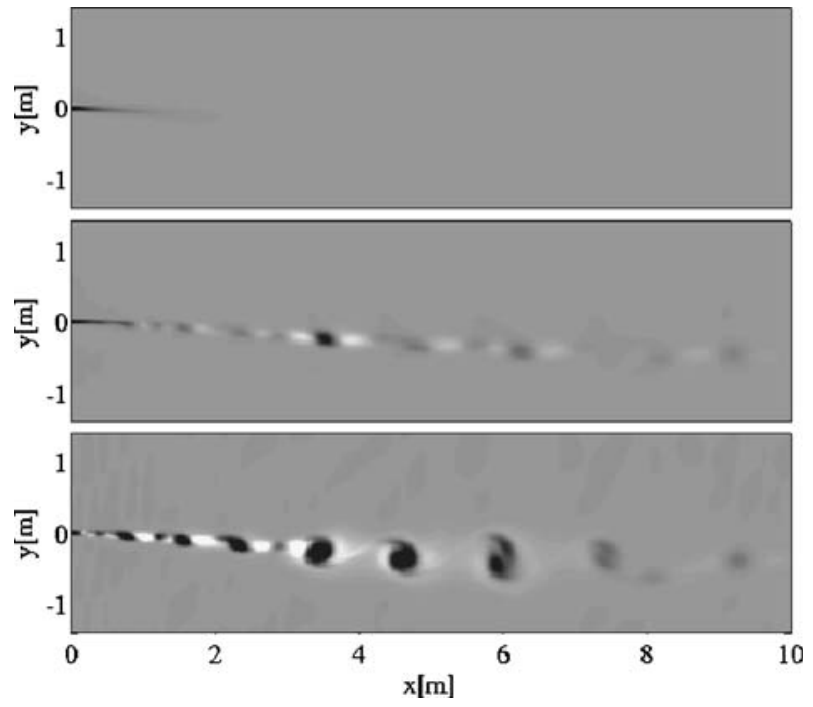

the center of the mixing layer shifts to the low-velocity side. The vorticity is reflecting the mean shear which decreases downstream where the mixing layer widens and the velocity difference decreases. Only with a sufficient level of disturbances the mixing layer forms clear eddy structures as seen in the middle and lower panel. A higher intensity at the inflow boundary leads to high intensity vortices that are generated further upstream. With the formation of turbulence structures also the merging of vortices can be observed. The stronger instabilities also lead to a somewhat wider mixing layer. At $x=8 \mathrm{~m}$ the mixing layer width for the cases shown in Fig. 3 are respectively $\delta=0.27 \mathrm{~m} ; \delta=0.32 \mathrm{~m}$ and $\delta=0.48 \mathrm{~m}$. It should be noticed here that the disturbances are still small with respect to the mean flow and subjected to the dissipation in accordance with (2) and (3).

While being advected by the mean flow the inflow disturbances will gradually disappear with downstream distance due to viscous and frictional effects and the absence of a resolved boundary layer in which turbulent kinetic energy is produced. This already demonstrates the arbitrariness in prescribing the ambient turbulence level only at the inflow boundary.

The intensity of the large structures formed turns out to be almost proportional to the imposed level of disturbances. As seen in Fig. 4 the energy density levels at $5 \mathrm{~m}$ downstream have the same shape but differ only in proportion to the inflow disturbance level. Note that the peaks of the spectra slightly shift to the low frequency side if the energy level increases. This is consistent with a widening of the mixing layer. The effect on the widening is however limited. An increase of the energy levels with a factor hundred results in an increase in mixing layer width of less than a factor two. This suggests that the amplification by the shear layer can be considered as an almost linear process and that the assumptions made with the linear stability analysis of [33] are justified. In other words, the amplification of the initial disturbances by the shear-instabilities is virtually the same in cases shown in Fig. 4. 
Fig. 4 Spectra of energy densities of the lateral velocity component at $5 \mathrm{~m}$ downstream of the mixing layer apex obtained with a depth averaged simulation for three levels of spectral energy density supplied at the inflow boundary: $E_{\mathrm{per}}=0.01 \mathrm{c}_{f} \mathrm{U}^{2} \mathrm{D}$ dotted, $E_{\mathrm{per}}=0.1 \mathrm{c}_{f} \mathrm{U}^{2} \mathrm{D}$ solid, $E_{\mathrm{per}}=1.0 \mathrm{c}_{f} \mathrm{U}^{2} \mathrm{D}$ dashed

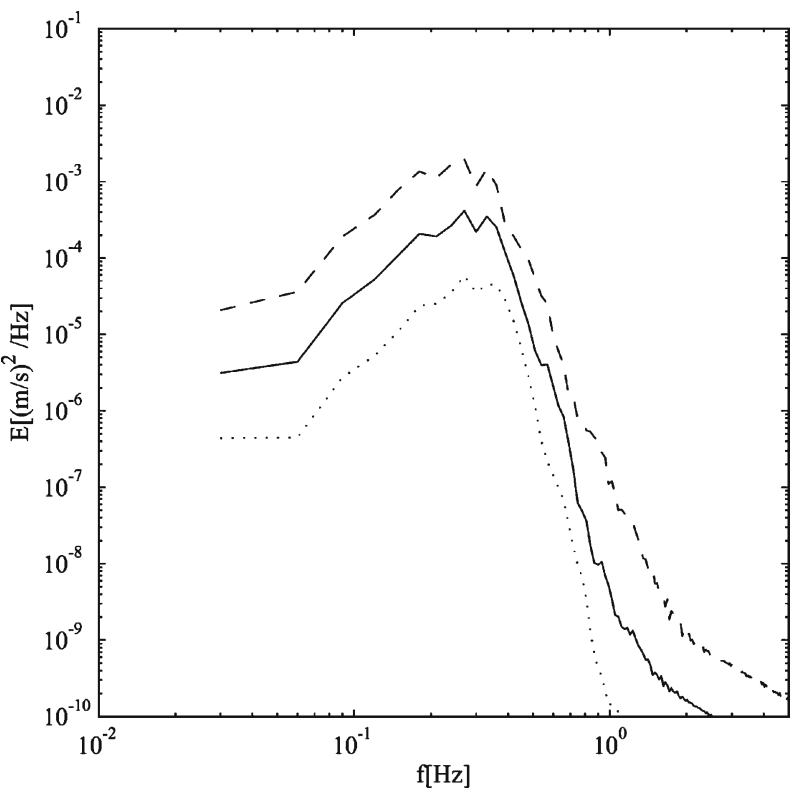

The intensity of the background quasi 2D turbulence is however determined fully empirically. In the next section the background quasi $2 \mathrm{D}$ turbulence will be determined, based on 3D-LES.

\subsection{Background quasi 2D turbulence}

In the previous section it was demonstrated that the development of the large scale structures depends on the level of disturbances imposed at the inflow boundary. In reality, these disturbances are not only present at the inflow boundary but throughout the whole domain as they are part of the turbulence in the bottom boundary layer. These fluctuations do contribute to the dissipative eddy-viscosity, but also form the seedlings from which larger structures can grow.

To determine the background quasi 2D turbulence, a 3D LES is carried out for a uniform channel flow in a wide domain: $80 D \times 80 D \times D$, see [34]. The results were checked with the DNS of the [21]. From the simulations the spectra of the quasi 2D turbulence were extracted. The spectra for the streamwise and transverse velocity are shown in Fig. 5. In order to take these fluctuations into account in the depth-averaged shallow water equations two steps are taken: (1) the fluctuations are mimicked by means of a kinematic simulation; (2) the fluctuations are implemented in the depthaveraged shallow water equations.

The fluctuating velocities $u_{k s}$ and $v_{k s}$ are derived for a flow field which is divergence-free, continuous in time and space [11], and is defined by:

$$
u_{k s}(x, y, t)=\frac{1}{N} \sum_{n=1}^{N} \sqrt{2} \sin \left(k_{x}^{n} U t+k_{y}^{n} V t+\theta^{n}\right) \hat{u}^{n} \sin \left(k_{x}^{n} x+k_{y}^{n} y+\phi^{n}\right),
$$



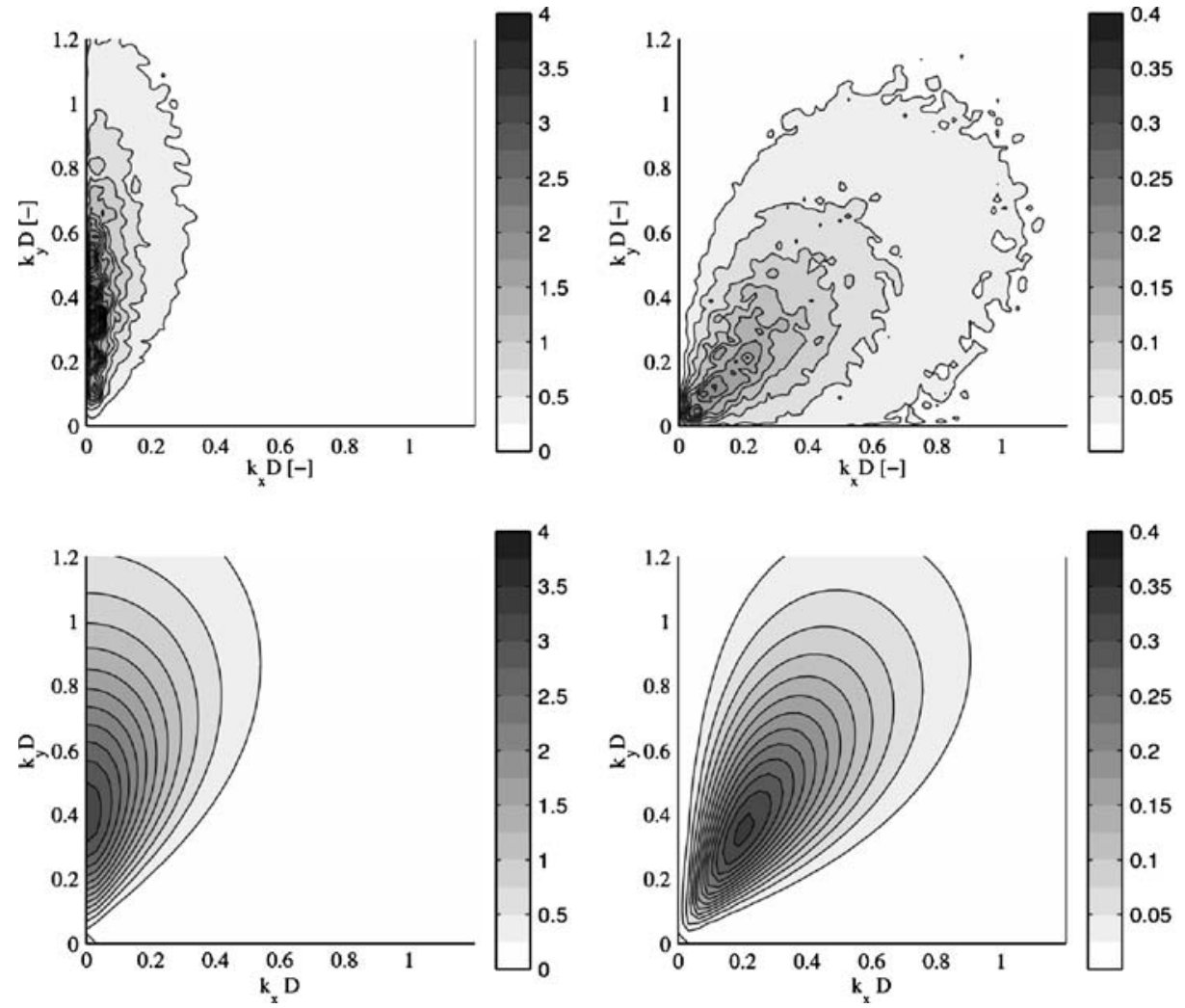

Fig. 5 Two-dimensional energy density spectra of the streamwise (left) and transverse (right) fluctuations of the depth averaged velocities. The spectra are based on the results of 3D-LES (top) and the results of the kinematic simulation (7) and (8) (bottom)

$$
v_{k s}(x, y, t)=-\frac{1}{N} \sum_{n=1}^{N} \sqrt{2} \sin \left(k_{x}^{n} U t+k_{y}^{n} V t+\theta^{n}\right) \hat{v}^{n} \sin \left(k_{x}^{n} x+k_{y}^{n} y+\phi^{n}\right),
$$

with: $k_{x}^{n}=\cos \left(\varphi^{n}\right) k^{n}, k_{y}^{n}=\sin \left(\varphi^{n}\right) k^{n}$, and $k^{n}=\sqrt{k_{x}^{n 2}+k_{y}^{n 2}}$.

Here $\phi^{\mathrm{n}}, \phi^{\mathrm{n}}$ and $\theta^{\mathrm{n}}$ are randomly chosen for each $n(N=200)$. The advection of the disturbance field is approximated by the constant streamwise and transverse velocities $U$ and $V$. In this paper we assume $U$ as the constant mean streamwise velocity in the center of the mixing layer and $V=0$. In future simulations, especially for complex flows, $U$ and $V$ should be able to vary in space and in time. Equations (5) and (6) should then be revisited, as this set will not be divergence free for gradients in the mean flow field $(\mathrm{U}, \mathrm{V})$. The amplitudes $\hat{u}^{n}$ and $\hat{v}^{n}$ can still be adjusted to the requirements for the specific turbulence properties. Important criteria in this respect 
are: the amplitude itself, typical correlation length, anisotropy and continuity. These requirements are empirically determined by:

$$
\begin{gathered}
\hat{u}^{n}\left(k_{x}^{n}, k_{y}^{n}\right)=\underbrace{\alpha u_{*}}_{\begin{array}{c}
\text { velocity } \\
\text { scaling }
\end{array}} \underbrace{\lambda\left|k^{n}\right| D \exp \left(-\lambda k^{n} D\right)}_{\begin{array}{c}
\text { length } \\
\text { scaling }
\end{array}} \underbrace{\left(\frac{k_{y}^{n}}{\left|k^{n}\right|}\right)^{\beta}}_{\text {anistropy }} \underbrace{\frac{k_{y}^{n}}{\left|k^{n}\right|}}_{\text {continuity }}, \\
\hat{v}^{n}\left(k_{x}^{n}, k_{y}^{n}\right)=\alpha u_{*} \quad \lambda\left|k^{n}\right| D \exp \left(-\lambda k^{n} D\right)\left(\frac{k_{y}^{n}}{\left|k^{n}\right|}\right)^{\beta} \frac{k_{y}^{n}}{\left|k^{n}\right|} .
\end{gathered}
$$

As the fluctuation stem from the bottom turbulence it is likely that the amplitude will scale with $\mathrm{u}_{*}$. The length scaling part is determined empirically with a linear increase for small wavenumbers and an exponentional decay to higher wavenumbers. The anisotropy part is also determined empirically. The continuity part directly follows from the continuity requirement. The formulation was based on the 2D spectra of the 3D-LES, see Fig. 5.

The free parameters $\alpha, \beta$ and $\lambda$ are fitted to data obtained from the above mentioned fully-3D LES of a straight channel flow. Given the constraints of the ks-formulation optimal results were obtained using: $\alpha=1.3, \beta=3.0$ and $\lambda=0.3$ in (7) and (8). For the proper coverage of the spectra $N=200$ modes were taken at equidistant wavenumber intervals. (for more details, see [34]). Figure 5 shows the two-dimensional energy density spectra as obtained after depth-averaging of the 3D-LES result and as generated using the kinematic simulation of (7) and (8). It should be noted that with the large domain the wave-number spectrum can be calculated down to very small values of $k D$. The raggedness in the graph in the left panel is due to the small number of independent samples (100) used for the determination of the 2D-spectrum. It shows the rather isotropic distribution of the lateral velocity component over both directions in wave-number space and the anisotropic distribution of the streamwise component.

For the determination of the additional source terms in the depth-averaged shallow water equations, the linearized shallow water equations for the velocity fluctuations were used as starting point:

$$
\begin{gathered}
\frac{\partial u^{\prime}}{\partial t}+U \frac{\partial u^{\prime}}{\partial x}=-\frac{1}{\rho} \frac{\partial p^{\prime}}{\partial x}-\frac{2 c_{f} U}{D} u^{\prime}+v_{t} \nabla^{2} u^{\prime}, \\
\frac{\partial v^{\prime}}{\partial t}+U \frac{\partial v^{\prime}}{\partial x}=-\frac{1}{\rho} \frac{\partial p^{\prime}}{\partial y}-\frac{c_{f} U}{D} v^{\prime}+v_{t} \nabla^{2} v^{\prime},
\end{gathered}
$$

where the prime denotes the fluctuations with respect to the mean value $(u=$ $U+u^{\prime}$ ). It is noted that these equations hold for uniform flow (no mean transverse velocity) and that $U \gg u^{\prime}$ and $U \gg v^{\prime}$. It was hypothesized that in a uniform channel flow the additional kinematic forcing terms in the depth-averaged shallow water equations should equal the sink terms (bottom friction and turbulent shear stress) 
in the shallow water equations for the velocity fluctuations. The resulting depthaveraged shallow water equations then read:

$$
\begin{gathered}
\frac{\partial \tilde{u}}{\partial t}+u \frac{\partial u}{\partial x}+v \frac{\partial u}{\partial y}=-\frac{1}{\rho} \frac{\partial \tilde{p}}{\partial x}-\frac{c_{f}}{D} u \sqrt{u^{2}+v^{2}}+v_{h o r} \nabla^{2} u+\underbrace{\frac{2 c_{f} U}{D} u_{k s}-v_{t} \nabla^{2} u_{k s}}_{\text {backscatter }}, \\
\frac{\partial v}{\partial t}+u \frac{\partial v}{\partial x}+v \frac{\partial v}{\partial y}=-\frac{1}{\rho} \frac{\partial \tilde{p}}{\partial x}-\frac{c_{f}}{D} v \sqrt{u^{2}+v^{2}}+v_{h o r} \nabla^{2} v+\underbrace{\frac{c_{f} U}{D} v_{k s}-v_{t} \nabla^{2} v_{k s}}_{\text {backscatter }} .
\end{gathered}
$$

with $U$ the mean streamwise velocity. The added source terms on the right hand side represent the forcing by the kinematic simulation of the large scale contributions stemming from the bottom turbulence flow.

For the specific case of a straight open channel flow, a 2D simulation without the described model would result in a trivial stationary uniform solution for the mean flow. Without side walls no velocity gradients are resolved that could lead to the production of turbulent kinetic energy. Fluctuations that appear are solely due to the applied forcing. In order to check the performance of the forcing scheme a snapshot of the depth averaged velocity field resulting from the 3D LES is compared with the 2D simulation in Fig. 6; only a part of the total computational domain of 80 times 80 water depths is displayed. The fluctuating components show realistic patterns for the longitudinal and lateral velocity fluctuations. The anisotropy in the flow structures is clearly seen. Note that the typical length scales for the observed structures is much larger than the water depth while the amplitude is still a few percent of the mean flow velocity $[16,18]$. Although in many cases the water depth $D$ is considered as a proper measure for the most important macroscopic length scales in the flow, in the depth-averaged case here the scales exceeding $D$ play a key role, despite their small amplitude. For further use the method described in this section will be labelled as the ks-backscatter model. The term backscatter is used here to classify the model as a source term rather than to indicate the direction of energy transfer.

\subsection{The shallow mixing layer}

The above described and validated method is now applied to the depth-averaged modelling of a spatially developing mixing layer as depicted above. It is anticipated that the 3D-turbulence generated in the boundary layers of the two streams has the same spectral signature as that of a straight channel flow. Naturally it can not be expected that the same holds for the mixing layer. It should however be noted that the ks-backscatter model is supposed to only provide the controlled necessary disturbance level for excitation of 2D flow structures in the mixing layer. Equations (11) and (12) are solved numerically using the same computational grid as with the mixing layer as described in Section 2.2, i.e. a grid of 150 by 850 cells covering a domain of $3 \mathrm{~m}$ by $17 \mathrm{~m}$. For simplicity we use a constant, uniform advection velocity $U$ in the ks-backscatter model (5) and (6), equal to the value in the center of the mixing layer. Introducing the lateral shear of the mixing layer also in the ksbackscatter model would make the model unnecessary complicated with respect to the evolution of the structures. 
a
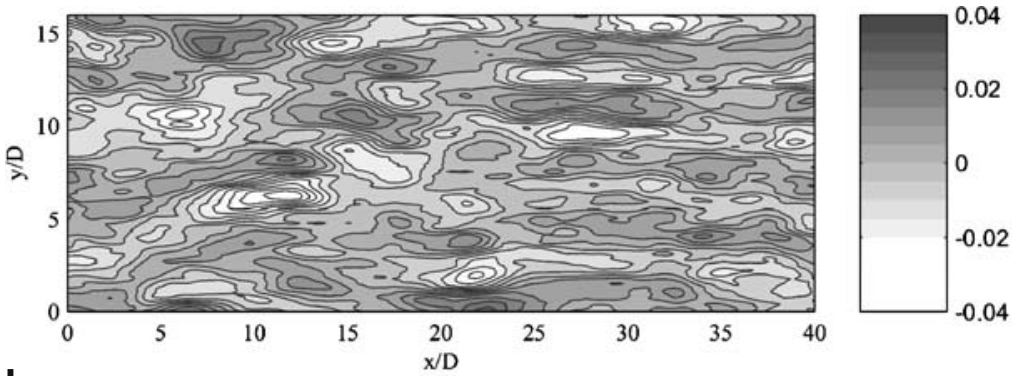

$\mathrm{u}_{3 \mathrm{D}-\mathrm{LES}}$

b
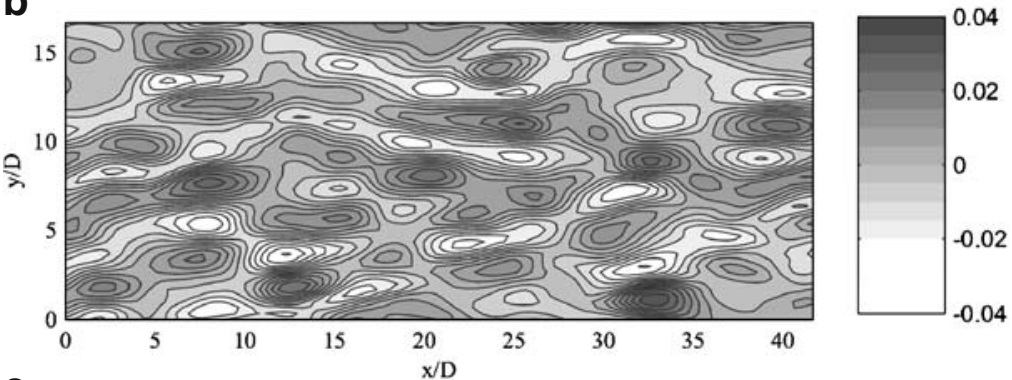

$\mathrm{u}_{2 \mathrm{D}-U R A N S}$

C
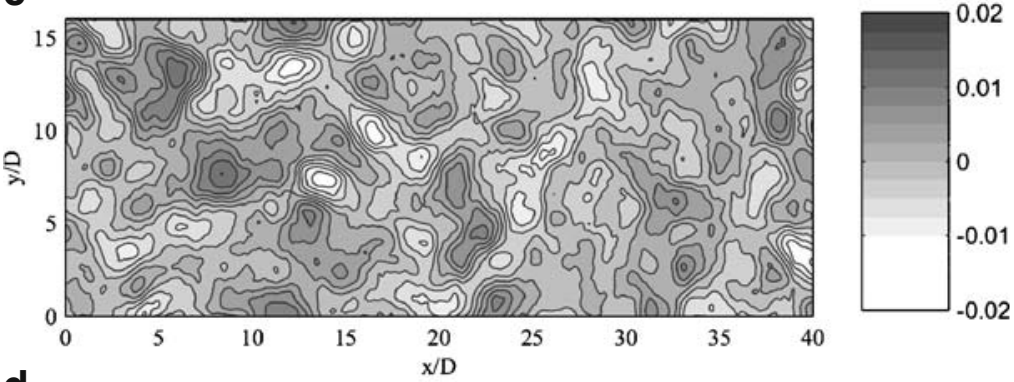

$\mathrm{V}_{3 \mathrm{D}-\mathrm{LES}}$
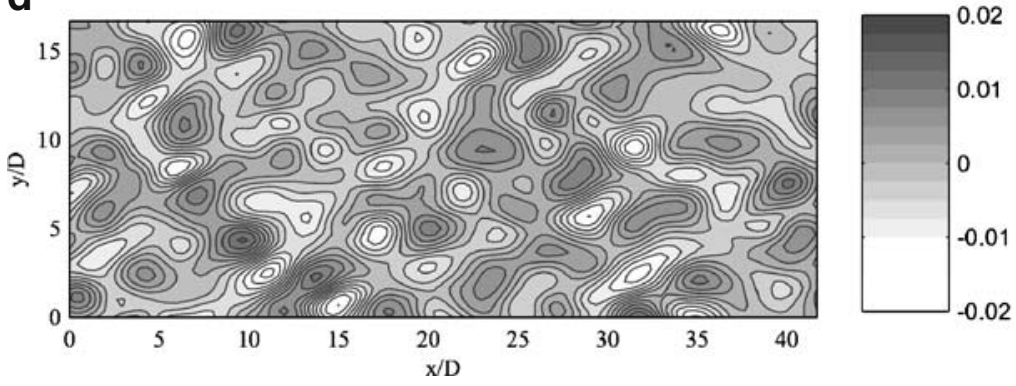

$\mathrm{V}_{2 \mathrm{D}-U R A N S}$

Fig. 6 Simulation results of instantaneous streamwise and transverse velocity fluctuations scaled with the mean velocity for a straight channel flow. a Depth averaged streamwise velocity fluctuations from the full 3D-LES. b Streamwise velocity fluctuations from the 2D URANS simulation with inclusion of the ks-backscatter model. c and $\mathbf{d}$ For the transverse velocity component

The results compare fairly well with the experimental data obtained from the freesurface motion, as far as the mean flow field is concerned (Fig. 7). The development of the mixing layer width and the lateral shift are well predicted. The turbulent 
a
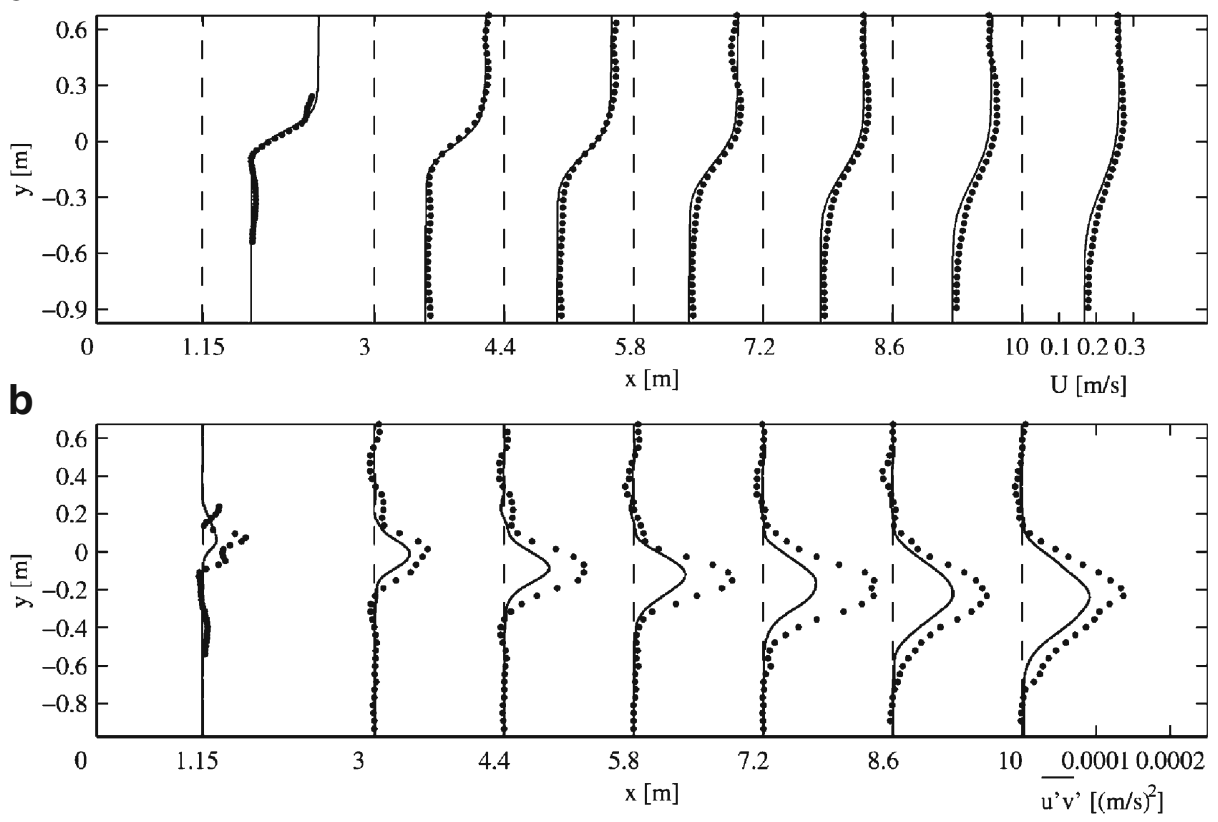

Fig. 7 Results of the URANS simulation with ks-backscatter (solid line) of the shallow mixing layer flow, compared with experimental data [34]. a mean streamwise velocity; b turbulent shear stress

shear stress is somewhat underestimated (Fig. 7). It is likely that $3 \mathrm{D}$ effects in the experiment, which are absent in the simulation, are causing part of this difference.

It should be noted that the measured data obtained at the free-surface are not fully representing the depth averaged motion. Experimental data of depth-averaged instantaneous flow fields are very difficult to obtain. The free-surface motion is therefore used here as an effective alternative containing at least the signatures of the dominant large-scale motion. Although the large-scale motion is highly correlated over the vertical [32] some characteristic 3D effects affect the data comparison. The vertical shear gives rise to elongated vortices that contribute more to the longitudinal fluctuations than the 2D more isotropic vortices in the simulation. Also possible secondary circulation, not included in the model, could play a role.

An important advantage of the proposed model is the representation of the resolved large horizontal structures. It is therefore useful to make a more quantitative validation with respect to the spectral distribution of the turbulent kinetic energy. Figure 8 shows a comparison between the simulations and the experiments. For the case shown, the frequency of maximum energy is well predicted indicating the proper dimension of the large eddies. The peak amplitudes are properly represented for the streamwise component but overestimated for the transverse velocities. It is no surprise that the high frequency part of the spectrum is considerably underestimated. That part of the spectrum reflects the energy cascade and subsequent dissipation which for the smaller scales is governed by $3 \mathrm{D}$ processes that are not resolved here. In the simulation, energy is removed from the turbulent motions through bottom friction acting on all scales with a high turbulent viscosity acting predominantly on the smallest resolved scales. With the limited resolution and the 2D dynamics 
a

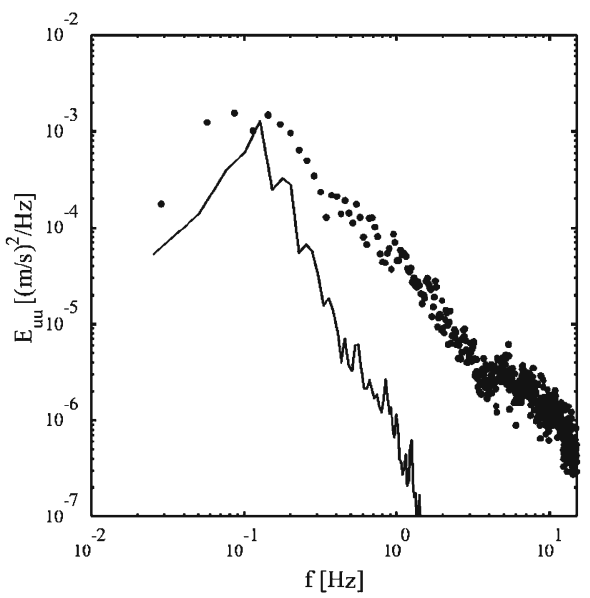

b

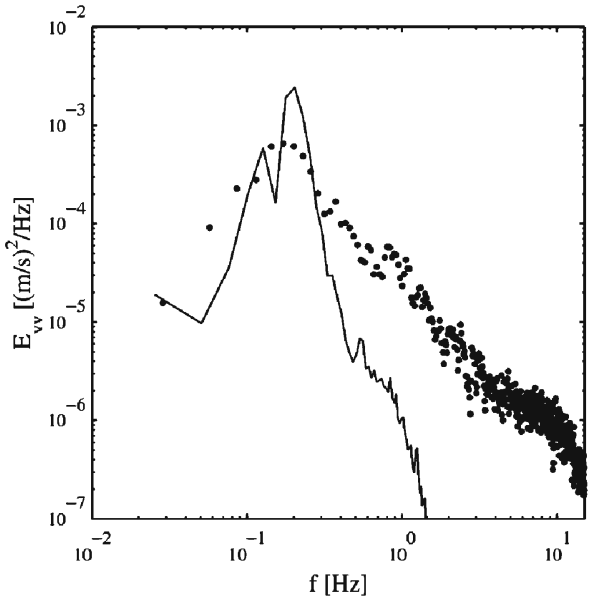

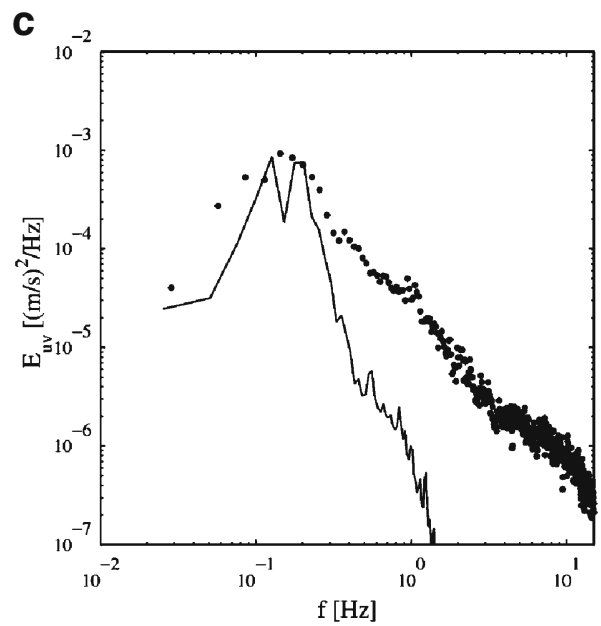

Fig. 8 Energy density spectra at $x=5.8 \mathrm{~m}$ in the center of the mixing layer of the resolved large scale fluctuations (lines) from the simulation with ks-backscatter, compared with experimental data (dots) from [34]

represented by the depth averaged model it is not surprising that the simulation yields large deviations in the energy transfer. In this respect a comparison with the spectra of depth averaged velocity measurements would be most appropriate despite the fact that the model lacks essential 3D physics. Nevertheless, as far as the large, energy containing eddies are concerned, the model works satisfactory and reveals the necessity of a backscatter model.

As we are interested in large eddy structures a comparison between experiments and the model should be made on eddy properties rather than turbulence intensities and spectra. However the stochastic nature of the flow makes it impossible to compare instantaneous flows, neither does it make sense to compare time averaged flow fields. In order to obtain an idea on the size, shape and intensity of typical vortex structures at a certain location, the method of conditional averaging is applied [25]. 

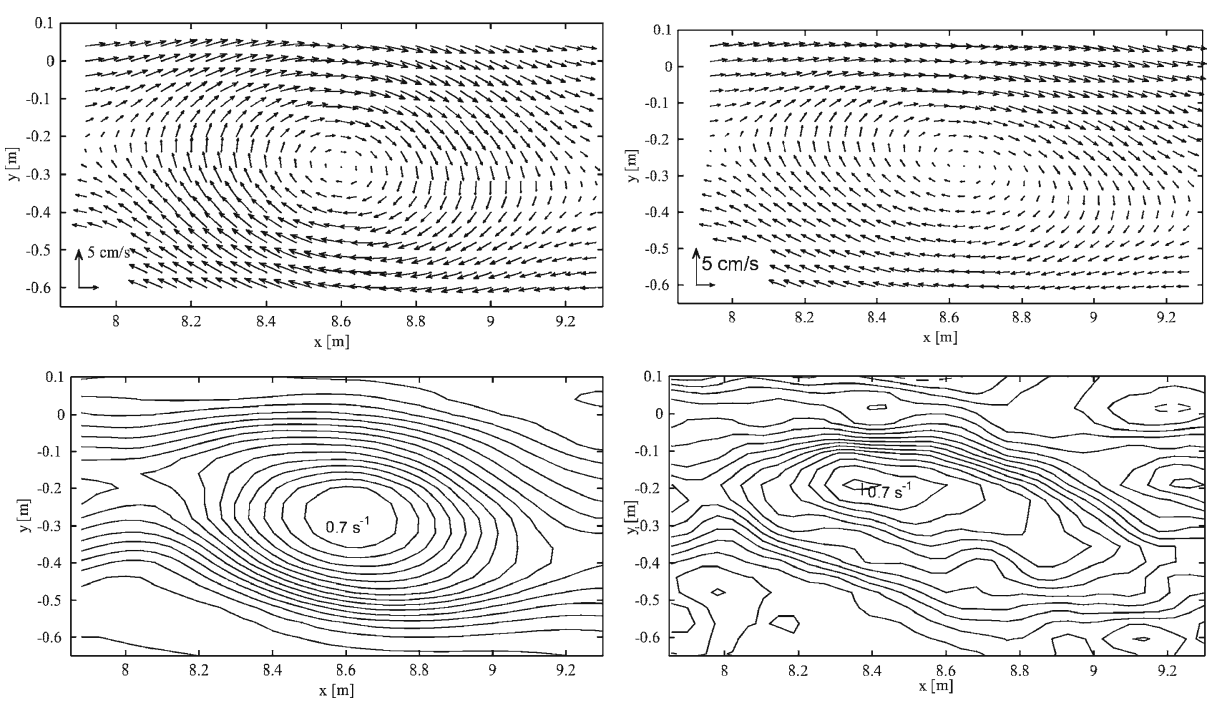

Fig. 9 Comparison of conditionally averaged eddies at $8.6 \mathrm{~m}$ downstream with a water depth of $67 \mathrm{~mm}$. Simulation data (left) and experimental data (right), vector fields (top) and vorticity contours (bottom)

Correlating a mask eddy of variable size and lateral position with time series of the flow field at a certain location, eddies can be identified and conditionally averaged. This method is applicable to both the experimental and the simulation data, allowing for a direct comparison between the results.

Figure 9 shows an example the conditionally averaged vortex at $8.6 \mathrm{~m}$ downstream. The vector fields clearly show a vortex structure of about $1 \mathrm{~m}$ length and $0.6 \mathrm{~m}$ width. The experimental eddy looks more elongated than the numerical one. This can also be observed in the representation of the structure in terms of the vorticity. Although the experimental data are more ragged and smaller in amplitude, the resemblance in amplitude and shape is reasonably good and it shows the level of detail at which the comparison can be made.

\section{Discussion}

The importance of the back-scatter model is evident for spatially developing shallow shear flows, where instabilities are triggered by 3D-turbulence by the shear in the vertical plane and where they are amplified to horizontal eddies by the shear in the horizontal plane. This is for example the case for lateral mixing layers as observed in compound channels (lateral variation in depth), lateral variations in roughness, sudden expansions, harbours, etc.

An aspect that has practical implications but has not received much attention here, is the effect of the large structures on the transport across the mixing layer. The shear stresses, denoting the transport of momentum, consists of contributions from the large eddies as well as the turbulent viscosity $v_{\text {hor }}$. From the growth rate of the mixing layer width only a slight dependency on the relative contribution of the large 
eddies is found, provided they occur with a significant amplitude. This is not just an aspect of shallow mixing layers. The universal growth behaviour of all kinds of deep mixing layers of uniform density is also an indication that the fluid properties and the intensity of large coherent structures are not of great influence, (see e.g. [3]).

Another aspect that results from this study is the comparison between depth averaged modelling results with measurements on a 3D flow configuration. In most cases it is impossible or takes too much effort to obtain sufficient data to determine an instantaneous value of the local depth-average velocity. A detailed verification is therefore not always feasible. In view of the characterization of large horizontal eddies a conditional averaging technique looks promising.

\section{Conclusions}

With the increased application of eddy resolving simulations (LES, URANS) to cases with a wide range of turbulent length/time scales, a growing need is observed for the representation of the non-resolved physics. For problems with a limited scale range, the implementation of a (standard) LES sub-grid model suffices for the inertial subrange. For wide range of scales problems URANS will be applied instead of LES and the limitations of the resolution then require more attention for the large, energy containing and often anisotropic turbulent motions. In shallow flows this typically comprises the three-dimensional turbulence generated in the vertical shear. When the dynamics of this 3D-turbulence is not or insufficiently resolved, the missing information can be replenished using a kinematic backscatter model. In devising this model it has become clear that in a straight open channel flow turbulent kinetic energy is present on length scales much larger than the water depth. In particular these turbulence structures are capable of triggering large scale instabilities in a shallow horizontal shear flow that lead to the formation of intense quasi two-dimensional eddies.

The work described above is still in progress and should be seen as the onset to a more extensive programme to improve shallow flow modelling tools. Only a relatively simple flow (shallow mixing layer) was considered, more complex flows have to be tested. A simplification was made by assuming a uniform advection velocity $[U=$ constant and $V=0$ in (5) and (6)]. A spatially and temporally varying advection velocity would be more general and has to be considered in the future. This will require revisiting (5) and (6) as these may introduce some divergence in the flow field. The parameters in the definition of the kinematic simulation are still empirical, although based on a 3D-LES of a channel flow. The validity of the parameters and their sensitivity to flow characteristics has to be further investigated.

At this stage the importance of this study lies in the notion that the large structures as found in unstable shear flows are influenced by the turbulent fluctuations stemming from other sources like for example bottom turbulence. In reducing the resolution or even the spatial dimensions of a simulation the lost information can have dramatic effects on the solution. This has obviously been well recognised and accounted for when it concerns the dissipative action of the small-scale turbulence, but not for their destabilizing effect. However, with instable flows much energy is available to amplify small-amplitude fluctuations into important large-scale flow structures. Their amplitude and spectral distribution should therefore be imple- 
mented in accordance with the underlying physics. In this study we focused on the interaction between the vertical boundary layer and the horizontal structures but other combinations can be thought of where the quasi two-dimensional flow structures (in a stratified or magnetically confined flow) are triggered by disturbances from a different origin.

Though not perfect, the proposed method provides at least a physically based method to efficiently include an important aspect of a shallow shear flow.

Acknowledgements This research is supported by the Technology Foundation STW, applied science division of NWO and the technology programme of the Ministry of Economic Affairs.

Open Access This article is distributed under the terms of the Creative Commons Attribution Noncommercial License which permits any noncommercial use, distribution, and reproduction in any medium, provided the original author(s) and source are credited.

\section{References}

1. Balaras, E., Benocci, C., Piomelli, U.: Two-layer approximate boundary conditions for large-eddy simulation. AIAA. J. 34(6), 1111-1119 (1996)

2. Batchelor, G.K.: Computation of the energy spectrum in homogeneous two-dimensional turbulence. Phys. Fluids Suppl. II, 233-239 (1969)

3. Brown, G.L., Roshko, A.: On density effects and large structure in turbulent mixing layers. J. Fluid Mech. 64, 775-816 (1974). doi:10.1017/S002211207400190X

4. Carati, D., Ghosal, S., Moin, P.: On the representation of backscatter in dynamic localization models. Phys. Fluids 7(3), 606-616 (1995). doi:10.1063/1.868585

5. Chu, V.H., Babarutsi, S.: Confinement and bed-friction effects in shallow turbulent mixing layers. J. Hydraul. Eng. 114, 1257-1274 (1988)

6. Domaradski, J.A., Saiki, E.M.: A subgrid-scale model based on the estimation of unresolved scales of turbulence. Phys. Fluids 9(7), 2148-2164 (1997). doi:10.1063/1.869334

7. Dracos, T., Giger, M., Jirka, G.H.: Plane turbulent jets in a bounded fluid layer. J. Fluid Mech. 214, 587-614 (1992). doi:10.1017/S0022112092002167

8. Elder, J.W.: The dispersion of marked fluid in turbulent shear flow. J. Fluid Mech. 5, 544-560 (1959)

9. Fischer, H.B., List, E.J., Koh, R.C.Y., Imberger, J., Brooks, N.H.: Mixing in Inland and Coastal Waters. Academic, New York (1979)

10. Flohr, P., Vassilicos, J.C.: A scalar subgrid model with flow structure for large-eddy simulations of scalar variances. J. Fluid Mech. 407, 315-349 (2000). doi:10.1017/S0022112099007533

11. Fung, J.C.F., Hunt, J.C.R., Malik, N.A., Perkins, R.J.: Kinematic simulation of homogeneous turbulence by unsteady random Fourier modes. J. Fluid Mech. 236, 281-318 (1992). doi:10.1017/S0022112092001423

12. Hamba, F.: A Hybrid RANS/LES simulation of turbulent channel flow. Theor. Comput. Fluid Dyn. 16, 387-403 (2003). doi:10.1007/s00162-003-0089-x

13. Hinterberger, C.: Three-dimensional and depth averaged large eddy simulation of shallow water flows. PhD-thesis University of Karlsruhe, Germany (in German) (2004)

14. Hinterberger, C., Fröhlich, J., Rodi, W.: Three-dimensional and depth-averaged largeeddy simulations of some shallow water flows. J. Hydraul. Eng. 133(8), 857-872 (2007). doi:10.1061/(ASCE)0733-9429(2007)133:8(857)

15. Hinterberger, C., Fröhlich, J., Rodi, W.: 2D and 3D turbulent fluctuations in open channel flow with $\operatorname{Re} \tau=590$ studied by large eddy simulation. Flow Turbul. Combust. 80, 225-253 (2008). doi:10.1007/s10494-007-9122-2

16. Jiménez, J.: The physics of wall turbulence. Physica A 263, 252-262 (1999). doi:10.1016/S03784371(98)00507-X

17. Johansson, P.S., Andersson, H.I.: Generation of inflow data for inhomogeneous turbulence. Theor. Comput. Fluid Dyn. 18, 371-389 (2004). doi:10.1007/s00162-004-0147-z

18. Kim, K.C., Adrian, R.J.: Very large-scale motion in the outer layer. Phys. Fluids 11(2), 417-422 (1999). doi:10.1063/1.869889 
19. Li, N., Balaras, E., Piomelli, U.: Inflow conditions for large-eddy simulations of mixing layers. Phys. Fluids 12(4), 935-938 (2000)

20. Mason, P.J., Thomson, D.J.: Stochastic backscatter in large-eddy simulations of boundary layers. J. Fluid. Mech. 242, 51-78 (1992). doi:10.1017/S0022112092002271

21. Moser, R.D., Kim, J., Mansour, N.N.: Direct numerical simulation of turbulent channel flow up to $\operatorname{Re}_{\tau}=590$. Phys. Fluids. 11(4), 943-945 (1999). doi:10.1063/1.869966

22. Piomelli, U., Ferziger, J., Moin, P., Kim, J.: New approximate boundary conditions for large eddy simulations of wall-bounded flows. Phys. Fluids A 1(6), 1061-1068 (1989)

23. Piomelli, U., Balaras, E., Pasinato, H., Squires, K.D., Spalart, P.R.: The inner-outer layer interface in large-eddy simulations with wall-layer models. Int. J. Heat Fluid Flow 24, 538-550 (2003). doi:10.1016/S0142-727X(03)00048-1

24. Pope, B.: Turbulent Flows. Cambridge University Press, UK (2000)

25. Scarano, F., Benocci, C., Riethmuller, M.L.: Pattern recognition analysis of the flow past a backward facing step. Phys. Fluids 11(12), 3808-3818 (1999). doi:10.1063/1.870240

26. Schumann, U.: Subgrid scale model for finite difference simulations of turbulent flows in plane channels and annuli. J. Comput. Phys. 18, 376-404 (1975). doi:10.1016/0021-9991(75)90093-5

27. Sous, D., Bonneton, N., Sommeria, J.: Turbulent vortex dipoles in a shallow water layer. Phys. Fluids 16(8), 2886-2898 (2004)

28. Spalart, P.R.: Strategies for turbulence modeling and simulations. Int. J. Heat Fluid Flow 21, 252-263 (2000). doi:10.1016/S0142-727X(00)00007-2

29. Temmerman, L., Hadziabdic, M., Leschziner, M.A., Hanjalic, K.: A hybrid two-layer URANSLES approach for large eddy simulation at high Reynolds numbers. Int. J. Heat Fluid Flow 26, 173-190 (2005). doi:10.1016/j.ijheatfluidflow.2004.07.006

30. Tennekes, H., Lumely, J.L.: A First Course in Turbulence. MIT-Press, Cambridge, MA (1972)

31. Uijttewaal, W.S.J., Booij, R.: Effects of shallowness on the development of free-surface mixing layers. Phys. Fluids 12(2), 392-420 (2000). doi:10.1063/1.870317

32. Uijttewaal, W.S.J., Tukker, J.: Development of quasi two-dimensional structures in a shallow free-surface mixing layer. Exp. Fluids 24, 192-200 (1998). doi:10.1007/s003480050166

33. Van Prooijen, B.C., Uijttewaal, W.S.J.: A linear approach for the evolution of coherent structures in shallow mixing layers. Phys. Fluids 14(12), 4105-4114 (2002). doi:10.1063/1.1514660

34. Van Prooijen, B.C.: Shallow mixing layers. PhD-thesis Delft University of Technology, The Netherlands (2004)

35. Westbury, P.S., Dunn, D.C., Morrison, J.F.: Analysis of a stochastic backscatter model for the larg-eddy simulation of wall-bounded flow. Eur. J. Mech. BFluids 23, 737-758 (2004). doi:10.1016/j.euromechflu.2004.01.003 